\title{
Efectos del fuego sobre un bosquecillo de Polylepis australis (Rosaceae) en las montañas de Córdoba, Argentina
}

\author{
Effects of fire on a Polylepis australis (Rosaceae) woodland in the mountains of \\ Córdoba, Argentina
}

DANIEL RENISON ${ }^{1,3}$, ANA M. CINGOLANI ${ }^{2,3} \&$ RICARDO SUAREZ $^{3}$

${ }^{1}$ Cátedra de Ecología General, Facultad de Ciencias Exactas, Físicas y Naturales, Universidad Nacional de Córdoba, Casilla de Correo 122, 5000 Córdoba, Argentina; e-mail: drenison@ com.uncor.edu

${ }_{2}^{2}$ Instituto Multidisciplinario de Biología Vegetal, Universidad Nacional de Córdoba-Consejo Nacional de Investigaciones Científicas y Técnicas

${ }^{3}$ Proyecto Conservación y Reforestación de las Sierras de Córdoba, Argentina

\begin{abstract}
RESUMEN
El fuego es usado para transformar bosques en pastizales y estimular el rebrote de los pastos para aumentar su valor forrajero. Según algunos autores, este uso del fuego ha provocado una retracción de los bosques de Polylepis a sitios rocosos inaccesibles al fuego, mientras que otros autores postulan que su distribución es debida al ambiente favorable en las rocas y no a la acción del fuego. Aquí evaluamos el efecto relativo del fuego y el ambiente sobre la supervivencia, producción de semillas y crecimiento de Polylepis australis Bitter (Rosaceae) en un bosquecillo de las montañas de Córdoba. Realizamos un seguimiento de 74 individuos quemados y controles durante 3,5 años. La presencia de rocas tuvo una fuerte influencia en el porcentaje en que se quemaron los individuos. A su vez, el porcentaje en que se quemaron se relacionó negativamente con la supervivencia, producción de semillas y el crecimiento en altura. La producción de biomasa fue mayor cuando se quemaron en un porcentaje intermedio. Exceptuando la protección al fuego, la roca no tuvo ningún efecto positivo sobre las variables medidas. Estos resultados sugieren que la distribución actual de $P$. australis es al menos en parte debida al fuego. Para su conservación sugerimos minimizar los fuegos, desarrollar actividades con alto riesgo de incendio en sitios con alta proporción de roca y, si es posible, proteger los ejemplares expuestos al fuego con piedras.
\end{abstract}

Palabras clave: Polylepis australis, fuego, supervivencia y crecimiento, microhábitat.

\begin{abstract}
Fire is used to transform woodlands into grasslands and to promote the regrowth of grasses to increase their forage value. According to some authors, this use of fire has caused a retraction of Polylepis woodlands to rock outcrops inaccessible to fire, while other authors postulate that their distribution is due to the favorable environment they find on rocks and not due to the influence of fire. We evaluated the relative effects of fire and environment on survival, seed production and growth of Polylepis australis Bitter (Rosaceae). We studied 74 burnt and control individuals during 3.5 years. The presence of rocks had a great influence on the percentage of $P$. australis that was burnt. In turn, the percent which was burnt was negatively related to survival, seed production and growth in height. Biomass production was highest when they were burnt in intermediate percentages. Rock had no positive effect on the studied variables, except as a protection against fire. Our results suggest that $P$. australis distribution is at least in part due to fire. For its conservation we suggest fire reduction, restrict high fire risk activities to areas with a high proportion of rock and, when possible, protect individuals exposed to fire with stones.
\end{abstract}

Key words: Polylepis australis, fire, survival and growth, microhabitat.

\section{INTRODUCCIÓN}

El fuego es una herramienta muy utilizada para transformar bosques en pastizales y estimular el rebrote de los pastos con el fin de aumentar su valor forrajero (Anderson 1981, Pucheta et al. 1997, Reich et al. 2001). Sin embargo, las transformaciones mediadas por el fuego pueden traer como consecuencia la pérdida de algunos servi- cios ecológicos que brindan los bosques. Por ejemplo, los bosques pueden ser de importancia para reducir la erosión del suelo, aumentar la captación y retención de las precipitaciones, y servir como refugio para muchas especies de flora y fauna (Fjeldså \& Kessler 1996, Kelty 1997, Spies 1998). La presencia de parches de bosque y árboles aislados puede ser beneficiosa para las mismas actividades ganaderas ya que los árboles proveen de 
sombra al ganado y fertilizan los suelos (Puerto 1997, De Miguel 1999). Por otro lado, existen sistemas en los cuales las leñosas no son afectadas por el fuego, o incluso necesitan del mismo para mantener sus poblaciones (Glitzenstein et al. 1995, Whelan 1995, Changxiang et al. 1997, Fulé \& Covington 1998, Gilliam \& Platt 1999). Por ello, para lograr un paisaje deseado mediante el manejo del fuego, es importante conocer la respuesta al fuego de las especies involucradas.

Algunas leñosas tienen cierta resistencia al fuego debido a su corteza gruesa, a yemas de crecimiento protegidas por hojas, a la capacidad de rebrote de sus raíces o tallos menos dañados, o a que su altura aleja del fuego los tejidos más susceptibles (Janzen 1967, Gill 1981). El ambiente post-fuego puede favorecer un aumento de la productividad (Wallace 1966, Abbott \& Loneragan 1983), un incremento en la producción de flores y semillas (Lamont \& Runciman 1993) y el aumento de la germinación y establecimiento de plántulas (Whelan 1995). En el otro extremo, los individuos quemados de especies susceptibles mueren, y el mantenimiento de sus poblaciones depende exclusivamente de la resistencia de las semillas, o de la capacidad de recolonización del sitio desde sectores no afectados (Anderson 1981, Whelan 1995). El fuego puede tener un efecto variable sobre los individuos de una misma especie, dependiendo de la topografía, los gradientes ambientales, la frecuencia e intensidad, época del año, clima anterior y posterior, el tamaño de las plantas y la presencia o ausencia de herbívoros (Leigh \& Holgate 1979, Zedler et al. 1983, Cary \& Morrison 1995, Segura et al. 1998).

En las Sierras Grandes de Córdoba, Argentina, el fuego es una herramienta de manejo importante que se usa asociada a las actividades ganaderas, presentes en la zona a partir del siglo XVII (Díaz et al. 1994). Los fuegos de origen natural, en cambio, son virtualmente nulos (Miglietta 1994). Los bosques de la parte alta de las Sierras Grandes están dominados por la especie Polylepis australis Bitter (Rosaseae). Los bosques se encuentran en las grandes quebradas aunque existen pequeños bosquecillos o individuos aislados en quebradas menores, sitios rocosos, y a lo largo de arroyos (Cabido \& Acosta 1985). Un patrón similar se observa en los Andes tropicales para otras especies de Polylepis (Fjeldså \& Kessler 1996). Según la hipótesis antrópica de Ellenberg (1979), la distribución de estas especies se debe a la actividad humana, ya que los hábitats donde se encuentran son sitios protegidos del fuego, el ganado y la tala. En particular, las rocas actuarían como protección, evitando que resulten afectados por el fuego (Ellenberg 1979, Kessler \& Driesch
1993, Fjeldså \& Kessler 1996). Según la hipótesis ambiental, la distribución se debe a las condiciones ambientales más favorables de los hábitats donde se encuentra la especie, ya que son sitios protegidos de los fríos extremos, y la humedad en las grietas de las rocas es mayor (ver Fjeldså \& Kessler 1996).

En este trabajo evaluamos el efecto del fuego sobre la supervivencia, producción de semillas y crecimiento en relación a la roca, el microhábitat y a la altura de los individuos, con el fin de determinar el efecto relativo del fuego y el ambiente. De esta manera aportamos datos para poner a prueba las hipótesis antrópica y ambiental sobre la distribución de Polylepis (Fjeldså \& Kessler 1996). Los objetivos específicos son: (1) analizar los efectos que ejercen la proporción de roca, otras variables de microhábitat y la altura de los individuos de $P$. australis sobre el porcentaje de dosel quemado por el fuego, (2) determinar el efecto relativo del grado en que son quemados y las variables de microhábitat sobre la supervivencia, producción de semillas y crecimiento, y (3) discutir las posibles implicancias para el manejo y la conservación de la especie.

\section{MATERIALES Y MÉTODOS}

\section{Área de estudio y características del fuego}

El estudio se realizó desde octubre de 1997 hasta abril 2001 en el macizo de "Los Gigantes" (31 25'02" S, 6448'09”O), situado en el extremo norte de las Sierras Grandes de Córdoba, Argentina. El paisaje está compuesto por pajonales y céspedes (Cabido 1985, Acosta et al. 1992), interrumpidos por afloramientos graníticos con vegetación rupícola (Funes \& Cabido 1995), y bosquecillos de $P$. australis (Cabido \& Acosta 1985). La precipitación media anual es de 854 $\mathrm{mm}$, ocurriendo la mayor parte (el $83 \%$ ) en verano (entre octubre y marzo) (Renison et al. en prensa). La temperatura media anual es de $8{ }^{\circ} \mathrm{C}$ (Cabido 1985).

Los efectos del fuego se estudiaron aprovechando una quema del pajonal realizada el 18 de octubre de 1997, al inicio de la estación húmeda. Durante la estación seca previa llovió $69 \mathrm{~mm}$ entre abril y septiembre, lo cual representa un 48 $\%$ de la media para esos meses. La estación húmeda posterior al fuego (octubre a marzo) fue más lluviosa que la media $(748 \mathrm{~mm}$, que representan un $105 \%$ de la media para esos meses). Durante el fuego, no hubo viento, la temperatura ambiental fue de $10{ }^{\circ} \mathrm{C}$ y el cielo estaba cubierto por nubes altas. 
El área quemada incluyó una quebrada de aproximadamente media hectárea, a $2.100 \mathrm{~m}$ de altura y con una pendiente general de 30 grados. La quebrada contenía un bosquecillo abierto de $P$. australis (aproximadamente $15 \%$ de cobertura arbórea), y un estrato bajo de pajonal de 20 a 40 $\mathrm{cm}$ de altura, mezclado con saliencias rocosas de diverso tamaño. Las llamas del fuego oscilaron entre 10 y $80 \mathrm{~cm}$ de altura y se desplazaron pendiente arriba cubriendo $200 \mathrm{~m}$ en $25 \mathrm{~min}$. Los individuos de $P$. australis sólo ardieron cuando el fuego proveniente de la vegetación de hierbas y pastos que lo rodeaban les incidía por varios segundos. El fuego no pasaba de una rama o individuo de $P$. australis a otro sin la ayuda del fuego proveniente de otras especies más combustibles.

\section{Relevamiento de campo}

Dos días después del fuego se revisó el área, registrándose 52 ejemplares de $P$. australis parcial o totalmente quemados, que fueron numerados individualmente. Asimismo, se identificaron y numeraron 22 individuos controles ubicados en una pendiente adyacente al área quemada, con similar topografía y distribución de tamaños. Para todos los individuos $(\mathrm{n}=74)$ se estimaron visualmente (excepto cuando se indica lo contrario) las siguientes variables: (1) porcentaje de roca en un área circular rodeando al tronco, con límite a $30 \mathrm{~cm}$ del perímetro del mismo; (2) microhábitat, que incluyó las características del sustrato (0-5 $\mathrm{cm}$ de profundidad) por debajo de la copa: porcentaje de suelo (partículas menores a 2 $\mathrm{mm}$ ), grava fina ( 2 a $5 \mathrm{~mm}$ ), grava gruesa ( 5 a 200 $\mathrm{mm}$ ) y roca (más de $200 \mathrm{~mm}$ ), así como la pendiente (grados), y la exposición al sol (grados). Esta última variable representa el ángulo correspondiente al recorrido solar que ilumina directamente al ejemplar estudiado. En una llanura sin obstáculos el valor de exposición al sol sería 180 grados, mientras que en sitios con pendientes, afloramientos y bloques rocosos que sombrean al ejemplar estudiado, el valor es menor; (3) altura inicial (anterior al fuego), que varió entre $10 \mathrm{y}$ $260 \mathrm{~cm}$. Para los individuos quemados, la altura anterior al fuego se estimó en base al remanente de las ramas quemadas. Se utilizó una cinta métrica; (4) porcentaje del dosel quemado, en base a la proporción de ramas y hojas afectadas.

En abril de 1998, 1999, 2000 y $2001(6,18,30$ y 42 meses después del fuego), se hicieron las siguientes mediciones, que corresponden a la primera, segunda, tercera y cuarta temporada de crecimiento posterior al fuego: (1) supervivencia: se consideró que el ejemplar había muerto si estaba seco o había desaparecido del sitio, tal como describen Renison \& Cingolani (1998); (2) crecimiento: se estimó como la diferencia de altura entre el final y el inicio del período considerado. Las alturas se midieron desde la base del individuo hasta la yema de crecimiento más alta; (3) producción de biomasa: luego de la primera y segunda temporada de crecimiento, se midió en cada individuo el número total y el largo de cada brote (exceptuando 5 individuos ubicados en lugares inaccesibles para realizar esta medición). El crecimiento de la temporada se distinguió del crecimiento de la temporada anterior por la marcada diferencia en el grado de lignificación. La suma del largo de todos los brotes del año se utilizó como un estimador de la biomasa producida (Buech \& Rugg 1989); (4) la producción de semillas se registró para la primera, segunda y cuarta temporada, como presencia/ausencia, en base a signos como ramitas remanentes de las inflorescencias y semillas que quedan adheridas durante varios meses después de la fructificación de enero y febrero.

\section{Análisis estadísticos}

Para determinar cuáles son los factores del microhábitat y del individuo que más influyen sobre el porcentaje de dosel quemado se utilizaron regresiones múltiples "stepwise" (Afifi \& Clark 1984). Se consideraron todos los individuos afectados por el fuego $(\mathrm{n}=52)$ y ocho variables: (1) porcentaje de roca hasta $30 \mathrm{~cm}$ del tronco; (2) suelo, (3) grava fina, (4) grava gruesa, y (5) roca por debajo de la copa; (6) pendiente; (7) exposición al sol; (8) altura del individuo. El mismo procedimiento se utilizó para analizar los factores que influyen sobre el crecimiento y producción de biomasa. En este caso, se utilizaron todos los individuos (quemados + controles) que sobrevivieron hasta el final del estudio $(\mathrm{n}=65)$. A las ocho variables explicatorias consideradas arriba se les agregó el porcentaje de dosel quemado. Luego de un primer análisis exploratorio en el cual se observaron los gráficos de las variables independientes contra los residuales (Afifi \& Clark 1984), se decidió incluir también la variable "porcentaje de dosel quemado" elevada al cuadrado. Los factores que influyen sobre producción de semillas se analizaron por medio de regresiones logísticas (Afifi \& Clark 1984), considerándose las mismas variables explicatorias que para el crecimiento y producción de biomasa. Las regresiones que involucraron el crecimiento, la producción de biomasa, y la producción de semillas se realizaron para cada temporada por separado, y en el caso del crecimiento se realizó además una regresión para 
las cuatro temporadas juntas. En todas las regresiones se verificó que las variables significativas no estuvieran altamente correlacionadas entre sí ya que esto ocasionaría inestabilidades en el modelo de regresión (Afifi \& Clark 1984). Para determinar en qué medida la supervivencia fue afectada por el fuego, se comparó mediante la prueba $\mathrm{U}$ de Mann-Whitney el porcentaje de dosel que se había quemado entre los individuos muertos y los sobrevivientes al final del estudio.

\section{RESULTADOS}

\section{Efectos sobre el porcentaje de dosel quemado}

El porcentaje de dosel quemado resultó menor en los individuos de $P$. australis rodeados por mayor proporción de roca a $30 \mathrm{~cm}$ del tronco (Fig. 1) y ubicados en los sitios con mayor exposición al sol. La influencia de la proporción de roca fue, sin embargo, mucho más importante que la influencia de la exposición al sol, ya que explicó un $47 \%$ de la varianza en el porcentaje de dosel quemado, mientras que la exposición al sol explicó sólo el 7 $\%\left(\mathrm{r}^{2}=0,55 ; \mathrm{n}=52 ; \mathrm{P}<0,05\right)$. La correlación entre ambas variables explicatorias no fue significativa $\left(r_{p}=0,15 ; n=52 ; \mathrm{P}>0,05\right)$.

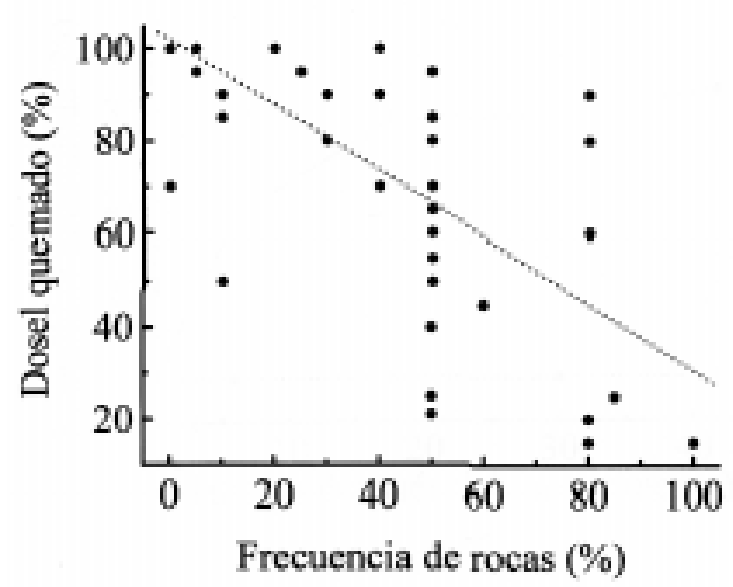

Fig. 1: Porcentaje de dosel de cada P. australis que resultó quemado en función del porcentaje de roca a $30 \mathrm{~cm}$ o menos del tronco. La recta (linea de puntos) fue calculada utilizando el modelo de regresión, y considerando la variable exposición al sol como una constante equivalente al promedio de dicha variable (111,35 grados).

Burnt percentage of each $P$. australis as a function of the percent of rock found at $30 \mathrm{~cm}$ or less from the trunk. The regression line (doted line) was calculated using the variable exposition to sun as a constant value equivalent to its average (111.35 degrees).

\section{Supervivencia}

Los individuos que sobrevivieron hasta el final del estudio $(n=65)$ habían sido quemados en un porcentaje significativamente más bajo que los individuos que murieron $(\mathrm{n}=9)(48,2 \%$ versus $98,9 \%, \mathrm{z}=-3,84 ; \mathrm{n}=74 ; \mathrm{P}<0,0001)$. El porcentaje de dosel quemado en los que murieron fue mayor al $90 \%$ en todos los casos (Fig. 2).

\section{Crecimiento}

En la primera temporada el crecimiento en altura fue afectado negativamente por el porcentaje de dosel quemado (Fig. 3A) (35\% de la varianza) y positivamente por la exposición al sol (3\% de la varianza) $\left(\mathrm{r}^{2}=0,38 ; \mathrm{n}=65 ; \mathrm{P}<0,05\right)$. En la segunda temporada el crecimiento fue afectado positivamente por la altura inicial $(25 \%$ de la varianza) $\left(\mathrm{r}^{2}=0,25 ; \mathrm{n}=65 ; \mathrm{P}<0,05\right)$. Para las tercera y cuarta temporadas, ninguna variable tuvo efectos significativos. Cuando se analizaron todas las temporadas juntas, el crecimiento fue afectado negativamente por el porcentaje de dosel quemado (Fig. 3B) (25\% de la varianza) y positivamente por la altura inicial $(15 \%$ de la varianza) $\left(\mathrm{r}^{2}=0,40 ; \mathrm{n}=65 ; \mathrm{P}<0,05\right)$. La relación entre el porcentaje de dosel quemado y el crecimiento fue cuadrática, indicando un incremento del efecto negativo a medida que aumentó el porcentaje de quemado (Fig. 3). De las variables explicatorias seleccionadas, la única correlación

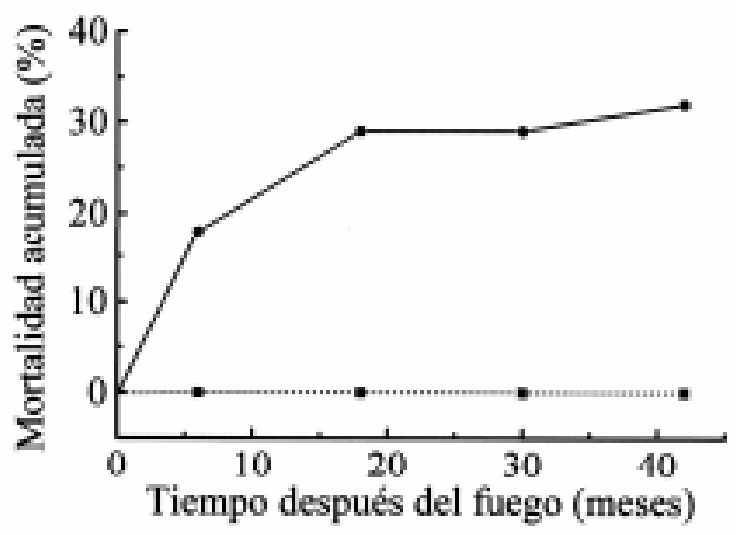

Fig. 2: Porcentaje de mortalidad acumulada para individuos de $P$. australis quemados en una proporción igual o mayor al $90 \%$ (linea continua) $(\mathrm{n}=28)$ y menor al $90 \%$ (linea de puntos) $(\mathrm{n}=$ 46).

Accumulated mortality, in percentage, for individuals of $P$. australis that were burnt $90 \%$ or more line (solid line) $(\mathrm{n}=$ $28)$ and less than $90 \%$ line (doted line) $(\mathrm{n}=46)$. 
significativa encontrada fue entre la exposición al sol y el porcentaje de dosel quemado, pero con un coeficiente de correlación bajo $\left(\mathrm{r}_{\mathrm{p}}=0,37 ; \mathrm{P}<\right.$ $0,01 ; \mathrm{n}=65)$. La disminución inicial de la altura (Fig. 3A) fue ocasionada por la muerte de las ramas más altas y finas y el rebrote de las ramas más bajas y gruesas incluyendo la base del tronco. Durante las cuatro temporadas, los individuos no quemados crecieron en promedio $40 \mathrm{~cm}$, mientras

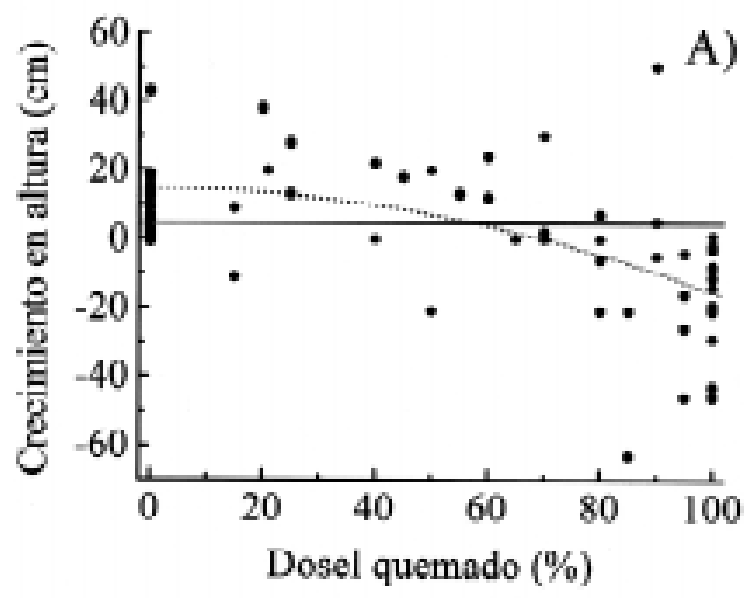

que los individuos quemados más del $80 \%$ crecieron sólo $10 \mathrm{~cm}$ (Fig. 3B).

\section{Producción de biomasa}

En la primer temporada, la producción de biomasa fue afectada de forma cuadrática por el porcentaje de dosel quemado (21\% de la varianza), con un

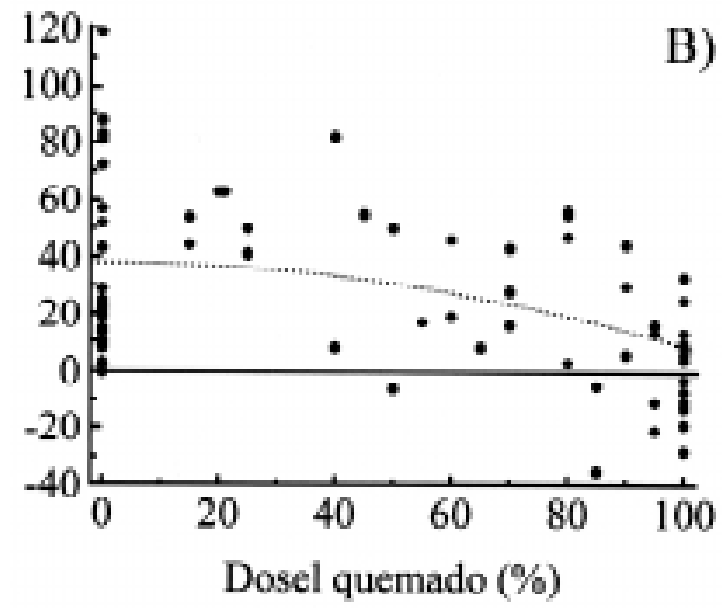

Fig. 3: Relación entre el porcentaje del dosel quemado para cada P. australis y su crecimiento en altura: (A) después de la primera temporada de crecimiento luego del fuego y (B), después de cuatro temporadas de crecimiento. En ambos casos la línea punteada representa la curva ajustada, calculada utilizando valores iguales al promedio para las restantes variables de la regresión múltiple (exposición al sol: 106,23 grados; altura inicial: $77,8 \mathrm{~cm} ; \mathrm{n}=65$ ).

Relation between the burnt percentage of each $P$. australis and its growth in height: (A) after the first post-fire growing season and, (B) after 4 growing seasons. In both cases the dashed line represents the fitted curve, calculated by using the average value for all other variables of the multiple regression (sun exposure: 106.23 degrees; initial height: $77.8 \mathrm{~cm} ; \mathrm{n}=65$ ).
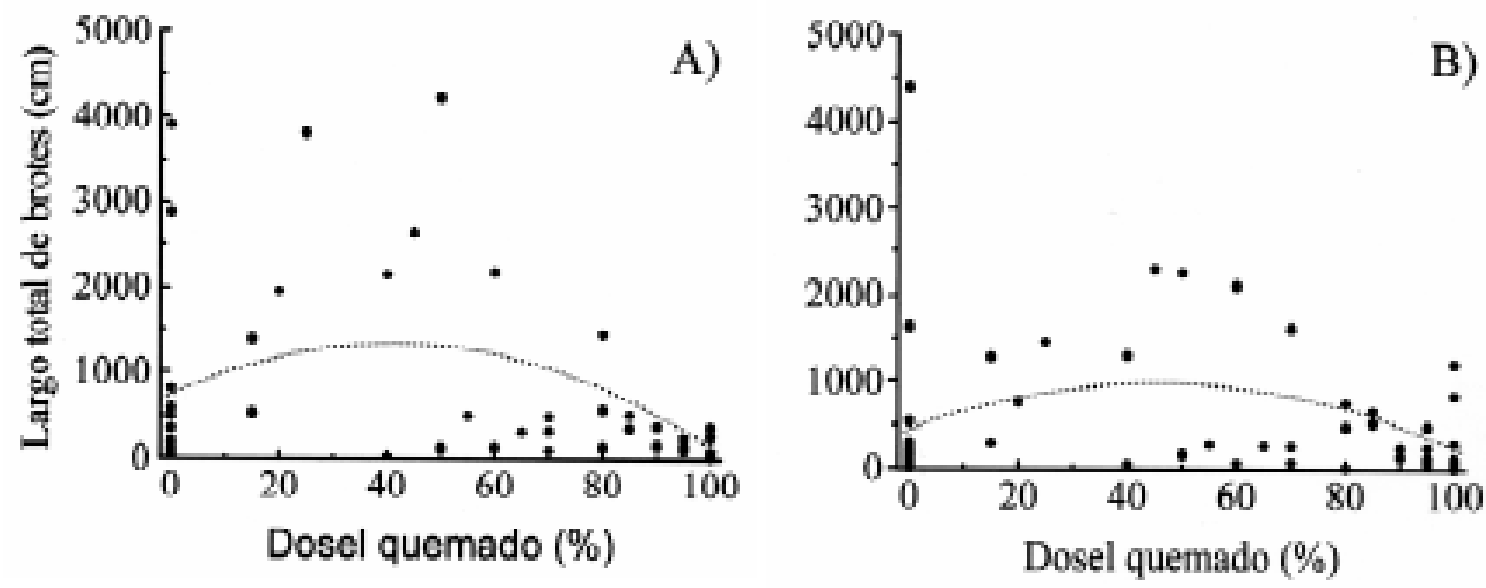

Fig. 4: Crecimiento en biomasa en la primera (A) y segunda (B) temporada de crecimiento en función del porcentaje de dosel quemado para cada $P$. australis. La línea punteada representa el valor ajustado según la regresión múltiple, calculado considerando valores iguales al promedio para las restantes variables (exposición al sol: 106,02 grados; pendiente: 32,97 grados; altura inicial: 79,83 cm; $\mathrm{n}=60$ ). Biomass growth in the first (A) and second (B) growing season as a function of burnt percentage of each $P$. australis. The dashed line represents the fitted curve, calculated by using the average value for all other variables of the multiple regression (exposition to sun: 106,23 degrees; slope: 32,97 degrees; initial height: 79,83 cm; $\mathrm{n}=60$ ). 
máximo de producción para los individuos quemados entre 40 y $50 \%$ (Fig. 4A). Adicionalmente, la producción fue afectada positivamente por la altura (25\% de la varianza) y el sol (4\% de la varianza), mientras que la pendiente afectó negativamente $\left(12 \%\right.$ de la varianza) $\left(r^{2}=0,62 ; n=60\right.$; $\mathrm{P}<0,05)$. En la segunda temporada la producción de biomasa también fue afectada de forma cuadrática por el porcentaje de dosel quemado (12\% de la varianza) (Fig. 4), y además fue afectada positivamente por la altura (12\% de la varianza), y negativamente por la pendiente (10 $\%$ de la varianza) $\left(\mathrm{r}^{2}=0,34 ; \mathrm{n}=60 ; \mathrm{P}<0,05\right)$. Las correlaciones significativas entre las variables explicatorias fueron bajas (entre la exposición al sol y el porcentaje de quemado $r_{p}=0,38 ; n=60$; $\mathrm{P}<0,01$; y entre la pendiente y altura del individuo $\left.r_{p}=0,30 ; n=60 ; P<0,05\right)$.

\section{Producción de semillas}

En la primera temporada la producción de semillas estuvo afectada positivamente por la altura (21\% de la varianza), negativamente por el porcentaje de grava fina $\left(\mathrm{r}^{2}=0,14 ; \mathrm{n}=65 ; \mathrm{P}<0,05\right)$ y el porcentaje de dosel quemado (4\% de la varianza). En segunda temporada y la cuarta temporada estuvo afectada positivamente por la altura (25\% de la varianza). La única correlación significativa entre las variables explicatorias fue baja (entre altura y porcentaje de grava fina: $r_{p}=$ $-0,32 ; \mathrm{n}=65 ; \mathrm{P}<0,01)$.

\section{DISCUSIÓN}

En las Sierras de Córdoba la mayoría de los fuegos son producidos al final de la estación seca (Miglietta 1994) en áreas con libre acceso al ganado (Pucheta et al. 1997) similares a la de nuestro estudio. Por ello consideramos que las condiciones en que se produjo el fuego fueron típicas de la zona y que los resultados de nuestro estudio son extrapolables a gran parte de los fuegos producidos en ambientes similares.

\section{Comparación con otras especies}

La supervivencia del $70 \%$ de los individuos de $P$. australis que se quemaron casi completamente (Fig. 2), define la especie como parcialmente resistente al fuego, y su capacidad de rebrotar la define como una especie resiliente (Rowe 1983, Whelan 1995). La resistencia de la especie posiblemente está dada por su poca combustibilidad y a su corteza en forma de finas láminas. La corteza de Polylepis aísla al tronco de las temperaturas ambientales extremas (Hensen 1994) y es probable que cumpla una función similar ante el fuego. Su resiliencia estaría dada por su capacidad de compensar la biomasa aérea perdida mediante el rebrote desde las ramas gruesas y la base de los troncos menos afectados por el calor. Este rebrote sugiere que el fuego contribuye a la arbustización de los ejemplares quemados, tal como se encontró Kessler \& Driesch (1993) en otras especies de Polylepis. El incremento de la producción de biomasa en los ejemplares de $P$. australis cuyo dosel fue moderadamente quemado puede deberse a varios factores. Entre ellos está el aumento de la temperatura del suelo debido a su oscurecimiento postfuego, el aumento de la disponibilidad de nutrientes, la disminución de la competencia de las plantas que crecen debajo de los árboles, el raleo causado por el fuego, o la persistencia de los sistemas radicales intactos que sustenten el crecimiento de estructuras aéreas disminuidas (Wallace 1966, Adams \& Anderson 1978, Whelan 1995).

Si bien $P$. australis puede clasificarse como una especie resiliente debido a su capacidad de rebrote, es de destacar que su resiliencia es moderada si se las compara con otras especies, como por ejemplo Banksia attenuata, cuyos individuos sobreviven gracias al rebrote en más del 99,5\% de los casos, cuando el intervalo entre fuegos es entre 5 y 40 años (Enright et al. 1998).

\section{Hipótesis de Ellenberg}

Las rocas actuaron protegiendo a $P$. australis del fuego, permitiendo que los individuos protegidos sobrevivieran en su totalidad. Los individuos que se quemaron, en cambio, tuvieron una pequeña probabilidad de morir, produjeron menos semillas, se arbustizaron y redujeron su estatura. Se sabe que los fuegos son frecuentes en la zona (Cabido \& Acosta 1988, observación personal de los autores) y los ejemplares sin protección de roca soportan varios incendios durante sus vidas. Prueba de ello son las marcas de fuego que se encuentran en muchos ejemplares de $P$. australis y troncos muertos (observación personal de los autores). Además, a largo plazo el fuego produce pérdida de suelos y con ello disminuye el contenido de nutrientes y la capacidad de almacenar agua (Kennard \& Gholz 2001, Reich et al. 2001). Los individuos de $P$. australis aislados en sitios muy degradados tienen semillas menos viables (Renison \& Cingolani 1998) y los plantines crecen menos que en sitos con suelos bien conservados (Renison et al. en prensa). Si bien $P$. australis es una especie moderadamente resistente y 
resiliente al fuego, los fuegos recurrentes tendrían efectos negativos sobre sus poblaciones no asociadas a la protección con rocas. En consecuencia, nuestros datos apoyan la hipótesis de Ellenberg (1979) que postula que la asociación de Polylepis con ciertos hábitats más rocosos se debe a la influencia del fuego.

Los principales argumentos en contra de la hipótesis antrópica de Ellenberg (1979) resumidos en Fjeldså \& Kessler (1996) y que son aplicables a nuestra zona de estudio son la estrecha asociación entre $P$. australis y ciertos ambientes especiales, la resistencia de la especie al fuego, y su capacidad de rebrote. Sin embargo, todos estos argumentos pueden ser refutados en parte. $P$. australis habita en sitos con pendientes pronunciadas, que además generalmente son más rocosos, húmedos y sombreados. Sin embargo, nuestros resultados indican que crece mejor en sitios soleados y con poca pendiente. Por otro lado, exceptuando la protección contra el fuego, la roca no tuvo ninguna influencia favorable sobre las medidas de crecimiento, ni producción de biomasa y semillas. Debido a que nuestro estudio fue realizado en un hábitat de quebrada, no podemos descartar completamente la hipótesis ambiental. Sería de interés estudiar los efectos relativos del fuego y ambiente sobre el crecimiento en sitios más xéricos, tales como las zonas de altiplano y las cumbres de lomas, donde la falta de agua podría ser más limitante para $P$. australis y por ende su asociación con las rocas más necesaria, incluso sin la presencia de fuego. El efecto diferencial del fuego con respecto al ambiente ha sido documentado en este trabajo y en otras regiones por varios autores (e.g., Whelan 1995, Pyne et al. 1996, Segura et al. 1998).

El hecho de que los individuos de $P$. australis más expuestos al sol se quemen menos fue un resultado inesperado. El sol además influyó positivamente en el crecimiento. Nuestra explicación, es que los primeros P. australis en rebrotar luego del invierno fueron aquellos ubicados en sitios soleados (observación personal) donde presumiblemente las temperaturas son mayores. En consecuencia, cuando se produjo el fuego los ejemplares de $P$. australis ubicados en sitios más soleados habrían estado más verdes y por lo tanto menos proclives a quemarse.

No encontramos relación entre la altura de los individuos y el porcentaje en que éstos fueron quemados por el fuego, al menos dentro del rango de alturas encontrado en nuestro estudio (máximo 2,6 m). Para otras especies de Polylepis, Kessler $\&$ Driesch (1993) encontraron que en sitios con mucha evidencias de quema había mayor proporción de árboles altos, sugiriendo que existe una relación entre la mayor altura y el escape al fuego. En nuestro caso, es probable que de haber encontrado en el área de estudio árboles mayores (existen $P$. australis de hasta al menos $8 \mathrm{~m}$ ) hubiéramos encontrado una relación significativa. De ser cierto que los $P$. australis más grandes escapan del fuego, posiblemente si el intervalo entre un fuego y otro permite a la especie llegar a una altura de escape suficiente los mismos podrían ser menos afectados por los fuegos.

\section{Implicancias para el manejo y la conservación de P. australis}

En general, el fuego ejerce un efecto negativo sobre las poblaciones de $P$. australis. Por ello, si se desea conservar los bosques como tales y promover su regeneración sería importante minimizar la frecuencia de fuegos.

Cuando los individuos de $P$. australis se quemaron menos del $40 \%$ el efecto del fuego fue casi nulo. Por ello, en áreas con $P$. australis que tienen alto riesgo de incendios (e.g., zonas de acampe, senderos y carreteras), o que por razones de manejo de la vegetación es necesario prender fuego (e.g., para promover el rebrote de los pastizales) es importante tomar medidas para que los bosquecillos de $P$. australis se quemen lo menos posible. Debido a que el porcentaje de roca a 30 $\mathrm{cm}$ o menos del tronco tuvo un fuerte efecto protector contra el fuego, hacemos las siguientes sugerencias: (1) dentro de lo posible procurar que las áreas con alto riesgo de fuego sean en sitios con alta proporción de roca y (2) proteger los $P$. australis que no tienen protección con dos o tres rocas que tapen los pastos y hierbas circundantes en un radio de al menos $30 \mathrm{~cm}$ alrededor del tronco. Sugerimos investigar en el futuro otras formas de minimizar el porcentaje que se quema $P$. australis durante el fuego, como la elección de la época o condiciones climáticas apropiadas, la reducción de biomasa vegetal por debajo de $P$. australis, o en toda el área mediante pastoreo previo a la quema. Es de destacar sin embargo que los datos aquí presentados, así como los de Kessler $\&$ Driesch (1993) sugieren que el mejor manejo para la especie es evitar el fuego y las medidas arriba mencionadas serían para minimizar los efectos de los fuegos que son inevitables.

\section{AGRADECIMIENTOS}

Agradecemos a Duilio Schinner y todos los voluntarios que ayudaron con las tareas de campo. A Diego Gurvich, Gregorio Gavier y dos revisores 
anónimos por sus sugerencias y lectura crítica del manuscrito. A la asociación "Los Algarrobos", la Agencia Córdoba Ambiente S.E., el Club Andino Villa Carlos Paz y los participantes del proyecto Conservación y Reforestación de las Sierras Grandes por ayudar a financiar el trabajo. A la Whitney Laing Foundation por financiar la publicación de este artículo.

\section{LITERATURA CITADA}

ABBOTT I \& O LONERAGAN (1983) Influence of fire on growth rate, mortality and butt damage in Mediterranean forest of western Australia. Forest Ecology and Management 6: 139-153.

ACOSTA A, S DÍAZ, M MENGHI \& M CABIDO (1992) Patrones comunitarios a diferentes escalas espaciales en pastizales de las Sierras de Córdoba, Argentina. Revista Chilena de Historia Natural 65: 195-207.

ADAMS DE \& RC ANDERSON (1978) The response of a central Oklahoma grassland to burning. Southwestern Naturalist 23: 623-632.

AFIFI AA \& V CLARK (1984) Computer-aided multivariate analysis. Wadsworth, Inc., Belmont, California. 458 pp.

ANDERSON RC (1981) An evolutionary model summarizing the roles of fire, climate, and grazing animals in the origin and maintenance of grasslands: an end paper. En: Estes JR, RJ Tyrl \& JN Brunken (eds) Grasses and grasslands: sistematics and ecology: 297-308. University of Oklahoma Press, Norman, Oklahoma.

BUECH RR \& DJ RUGG (1989) Biomass relations of shrub components and their generality. Forest Ecology and Management 26: 257-264.

CABIDO M (1985) Las comunidades vegetales de la Pampa de Achala, Sierras de Córdoba, Argentina. Documents Phytosociologiques 9: 431-443.

CABIDO M \& A ACOSTA (1985) Estudio fitosociológico en bosques de Polylepis australis BITT. ("Tabaquillo") en las Sierras de Córdoba, Argentina. Documents Phytosociologiques 9: 385-400.

CABIDO M \& A ACOSTA (1988) Degradación en pastizales climáticos de las Sierras de Córdoba, Argentina. Zonación a partir del pastizal de Deyeuxia hieronymi en Pampa de Achala. Documents Phytosociologiques 11: 573-581.

CARY GJ \& DA MORRISON (1995) Effects of fire frequency on plant species composition of sandstone communities in the Sydney region: combinations of inter-fire intervals. Australian Journal of Ecology 20: 418-426.

CHANGXIANG L, PA HARCOMBE \& RG KNOX (1997) Effects of prescribed fire on the composition of woody plant communities in southeastern Texas. Journal of Vegetation Science 8: 495-504.

DE MIGUEL JM (1999) Naturaleza y configuración del paisaje agrosilvopastoral en la conservación de la diversidad biológica en España. Revista Chilena de Historia Natural 72: 547-557.
DÍAZ S, A ACOSTA \& M CABIDO (1994) Community structure in montane grasslands of central Argentina in relation to land use. Journal of Vegetation Science 5:483-488.

ELLENBERG H (1979) Man's influence on the tropical mountain ecosystems in South America. Journal of Ecology 67: 401-416.

ENRIGHT NJ, R MARSULA, BB LAMONT \& C WISSEL (1998) The ecological significance of canopy seed storage in fire-prone environments: a model for resprouting shrubs. Journal of Ecology 86: 960-973.

FJELDSA J \& M KESSLER (1996) Conserving the biological diversity of Polylepis woodlands of the highland of Peru and Bolivia. A Contribution to Sustainable Natural Resource Management in the Andes. Nordic Foundation for Development and Ecology, Copenhagen, Denmark. 250 pp.

FULÉ PZ \& WW CONVINGTON (1998) Spatial patterns of Mexican pine-oak forests under different recent fire regimes. Plant Ecology 134: 197-209.

FUNES G \& M CABIDO (1995) Variabilidad local y regional de la vegetación rupícola de las Sierras Grandes de Córdoba, Argentina. Kurtziana 24: 173 188.

GILL AM (1981) Adaptive responses of the Australian vascular plant species to fires. En: Gill AM, RH Groves \& I Noble (eds) Fire and the Australian biota: 243-272. Australian Academy of Science, Camberra, Australia.

GILLIAM FS \& WJ PLATT (1999) Effects of long-term fire exclusion on tree species composition and stand structure in an old-growth Pinus palustris (Longleaf pine) forest. Plant Ecology 140: 15-26.

GLITZENSTEIN J, WJ PLATT \& DR STRENG (1995) Effects of fire regime and habitat on the tree dynamics in north Florida longleaf pine savannas. Ecological Monographs 65: 441-476.

HENSEN I (1994) Estudios ecológicos y fenológicos sobre Polylepis besseri Hieron en la cordillera oriental boliviana. Ecología en Bolivia 23: 21-32.

JANZEN D (1967) Fire, vegetation structure, and the ant $\mathrm{x}$ acacia interaction in Central America. Ecology 48: 26-35.

KELTY MJ (1997) Silvicultural management of wildlife habitat. En: Smith DM, BC Larson, MJ Kelty \& PMS Ashton (eds) The practice of silviculture: 483-507. John Wiley, New York, New York.

KENNARD DK \& HL GHOLZ (2001) Effects of high- and low-intensity fires on soil properties and plant growth in a Bolivian dry forest. Plant and Soil 234: 119-129.

KESSLER M \& P DRIESCH (1993) Causas e historia de la destrucción de bosques altoandinos en Bolivia. Ecología en Bolivia 21: 1-18.

LAMONT BB \& HV RUNCIMAN (1993) Fire may stimulate flowering, branching, seed production and seedling establishment in two kangaroo paws (Haemodoraceae). Journal of Applied Ecology 30: 256-264.

LEIGH JH \& MD HOLGATE (1979) Responses of understory of forests and woodlands of the southern tablelands to grazing and burning. Australian Journal of Ecology 4: 25-45. 
MIGLIETTA S (1994) Patrón de ocurrencia de fuegos y su efecto sobre la vegetación en el bosque Serrano de Córdoba. Tesis de Maestría, Facultad de Ciencias Exactas, Físicas y Naturales, Universidad Nacional de Córdoba, Córdoba, Argentina. 60 pp.

PUCHETA E, M CABIDO \& S DÍAS (1997) Modelos de estados y transiciones para los pastizales de altura de las Sierras de Córdoba, Argentina. Ecotrópicos 10: 151-160.

PUERTO A (1997) La dehesa. Investigación y Ciencia 253: 66-73.

PYNE SJ, PL ANDREWS \& RD LAVEN (1996) Fire behavior. En: Pyne SJ, PL Andrews \& RD Laven (eds) Introduction to wildland fire: 46-127. Second edition. John Wiley \& Sons, Inc., New York, New York.

REICH PB, DW PETERSON, DA WENDIN \& K WRAGE (2001) Fire and vegetation effects on productivity and nitrogen cycling across a forest-grassland continuum. Ecology 82: 1703-1719.

RENISON D \& AM CINGOLANI (1998) Experiencias en germinación y reproducción vegetativa aplicados a la reforestación con Polylepis australis (Rosaceae) en las Sierras Grandes de Córdoba, Argentina. AgriScientia 15: 47-53.

RENISON D, AM CINGOLANI \& D SCHINNER (en prensa) Optimising restoration of Polylepis australis forests through reforestation: when, where and how to transplant seedlings to the mountains? Ecotropical Monographs.
ROWE JS (1983) Concepts of fire effects on plant individuals and species. En: Wein RW \& DA MacLean (eds) The role of fire in northern circumpolar ecosystems: 135-154. John Wiley, New York, New York.

SEGURA AM, M HOLMGREN, JJ ANABALÓN \& ER FUENTES (1998) The significance of fire intensity in creating local patchiness in the Chilean matorral. Plant Ecology 139: 259-264.

SPIES T (1998) Forest stand structure, composition, and function. En: Kohm KA \& JF Franklin (eds) Creating a forestry for the $21^{\text {st }}$ century: the science of ecosystem management: 11-30. Island Press, Washington, District of Columbia.

WALLACE WR (1966) Fire in the jarrah forest environment. Journal of the Royal Society of Western Australia 49: 33-44.

WHELAN RJ (1995) The ecology of fire. Cambridge University Press, Cambridge, United Kingdom. 346 pp.

ZEDLER PH, CR GAUTIER \& GS McMASTER (1983) Vegetation change in response to extreme events: the effect of a short interval between fires in California chaparral and coastal scrub. Ecology 64: 809-818. 